OPEN ACCESS

Edited by:

Keqiang Wu,

National Taiwan University, Taiwan

Reviewed by:

Yongjun Lin,

Huazhong Agricultural University,

China

Zhicheng Dong,

South China Botanical Garden (CAS),

China

*Correspondence:

Zonghua Wang

wangzh@fafu.edu.cn

Songbiao Chen

songbiao_chen@hotmail.com

Feng Wang

wf@fjage.org

tThese authors have contributed equally to this work.

Specialty section:

This article was submitted to Plant Genetics and Genomics, a section of the journal

Frontiers in Plant Science

Received: 11 May 2017 Accepted: 03 August 2017 Published: 21 August 2017

Citation:

Chen Z, Cheng Q, Hu C, Guo X, Chen Z, Lin Y, Hu T, Bellizzi M, Lu G,

Wang G-L, Wang Z, Chen S and Wang F (2017) A Chemical-Induced, Seed-Soaking Activation Procedure for Regulated Gene Expression in Rice. Front. Plant Sci. 8:1447. doi: 10.3389/fp/s.2017.01447

\section{A Chemical-Induced, Seed-Soaking Activation Procedure for Regulated Gene Expression in Rice}

Zaijie Chen ${ }^{1,2 t}$, Qianqian Cheng ${ }^{2 \dagger}$, Chanquan $\mathrm{Hu}^{2}$, Xinrui Guo², Ziqiang Chen², Yan Lin², Taijiao $\mathrm{Hu}^{2}$, Maria Bellizzi ${ }^{3}$, Guodong Lu' ${ }^{1}$, Guo-Liang Wang ${ }^{3}$, Zonghua Wang ${ }^{1 *}$, Songbiao Chen ${ }^{2 *}$ and Feng Wang ${ }^{2 *}$

${ }^{1}$ Fujian-Taiwan Joint Center for Ecological Control of Crop Pests, Fujian Agriculture and Forestry University, Fuzhou, China, ${ }^{2}$ Biotechnology Research Institute, Fujian Academy of Agricultural Sciences, Fuzhou, China, ${ }^{3}$ Department of Plant

Pathology, The Ohio State University, Columbus, $\mathrm{OH}$, United States

Inducible gene expression has emerged as a powerful tool for plant functional genomics. The estrogen receptor-based, chemical-inducible system XVE has been used in many plant species, but the limited systemic movement of inducer $\beta$-estradiol in transgenic rice plants has prohibited a wide use of the XVE system in this important food crop. Here, we constructed an improved chemical-regulated, site-specific recombination system by employing the XVE transactivator in combination with a Cre/loxP-FRT system, and optimized a seed-soaking procedure for XVE induction in rice. By using a gus gene and an hpRNAi cassette targeted for OsPDS as reporters, we demonstrated that soaking transgenic seeds with estradiol solution could induce highly efficient sitespecific recombination in germinating embryos, resulting in constitutive and high-level expression of target gene or RNAi cassette in intact rice plants from induced seeds. The strategy reported here thereby provides a useful gene activation approach for effectively regulating gene expression in rice.

Keywords: Oryza sativa, regulated gene expression, XVE system, DNA recombination, seed-soaking

\section{INTRODUCTION}

Inducible gene expression systems are powerful tools for basic research in plant functional genomics, and for plant biotechnology applications such as the deletion of selectable marker genes in transgenic plants (Moore et al., 2006). Over the last two decades, heat-inducible system (Zhang et al., 2003; Khattri et al., 2011; Nandy and Srivastava, 2012), and chemical-inducible systems responding to various inducers, such as glucocorticoid (Schena et al., 1991; Aoyama and Chua, 1997), copper (Mett et al., 1993; McKenzie et al., 1998), tetracycline (Weinmann et al., 1994; Bohner et al., 1999), herbicide safeners (De Veylder et al., 1997), ethanol (Caddick et al., 1998; Salter et al., 1998), ecdysone (Martinez et al., 1999), and $\beta$-estradiol (Bruce et al., 2000; Zuo et al., 2000) have been developed. Compared to constitutive gene expression driven by constitutive promoters, conditional gene expression controlled by the chemical-inducible systems has provided a more flexible approach for functional dissection of developmentally important genes, especially if the genes related to regeneration, growth, reproduction, or lethality.

Among the developed chemical-inducible systems, the estrogen receptor-based transactivator XVE (Zuo et al., 2000) appears to be one of the most reliable and efficient systems. XVE consists of a DNA-binding domain of the bacterial repressor LexA, a transactivating domain of herpes simplex 
virus VP16, and a human estrogen receptor. Characterization of the XVE system in Arabidopsis demonstrated that the target gene expression controlled by this system was highly induced and tightly regulated upon estradiol induction (Zuo et al., 2000). The XVE system has been applied to different plant species, including basal land plant moss (Kubo et al., 2013), flowering dicot (Zuo et al., 2000, 2001; Guo et al., 2003; Zhang et al., 2007; Schlücking et al., 2013) and monocot (Sreekala et al., 2005; Okuzaki et al., 2011) plants.

Rice (Oryza sativa L.) is one of the world's most important staple food crops and has been established as a model organism for the functional genomics of monocot plants. The availability of highly accurate genomic sequences of both indica (Yu et al., 2002) and japonica (International Rice Genome Sequencing Project, 2005) subspecies, and many other accumulated genomic and molecular resources (Kikuchi et al., 2003; Jung et al., 2008), has greatly promoted genomic and genetic studies in rice. The XVE system has been tested in rice calli to produce marker-free transgenic plants (Sreekala et al., 2005), and in both calli and seedlings to investigate target gene expression patterns (Okuzaki et al., 2011). The two reports showed that the XVE system is functional in regulating gene expression in rice calli and roots. In the leaves of intact plantlets treated with estradiol through root absorption, however, target gene induction was observed at a very low level (Okuzaki et al., 2011), becoming one of the major limitations for this system to be widely used in discovering gene function in rice.

In the present study, based on detailed investigation of XVEcontrolled gene expression patterns in calli, detached leaf pieces, and intact plantlets, we proposed that limited systemic movement of estradiol might be the key reason for low inducibility in intact rice plants. To meet the need for high-level target gene expression in intact rice plants, we further constructed an improved XVEcontrolled, site-specific recombination system and optimized the induction procedure for rice seeds. Experiments based on a reporter gus gene and an hpRNAi cassette targeted to a rice phytoene desaturase (OsPDS) gene showed that soaking transgenic seeds with estradiol solution could activate highly efficient site-specific recombination in germinating embryos, resulting in constitutive activation of the target gene or RNAi cassette in intact rice plants. Our method thereby provides a useful gene activation approach for functional genomics in rice.

\section{MATERIALS AND METHODS}

\section{Plasmid Construction}

Plant expression vectors pUX-GUS, pXCL-GUS, pXCLFGUS, and pXCLF-PDSi were constructed following standard molecular manipulation procedures (Sambrook and Russell, 2001), except that the TOPO cloning and the LR clonase reaction for constructing pXCLF-PDSi were carried out by following Gateway cloning protocol (Invitrogen, Carlsbad, CA, United States). All plasmids derived from PCR products or synthetic oligonucleotides were verified by sequencing. Details of plasmid construction are available in Supplementary Table S1.

\section{Rice Protoplast Transient Expression Assay}

Protoplasts were isolated from 2-week-old etiolated seedlings of rice, O. sativa $\mathrm{cv}$. Nipponbare. PEG-mediated protoplast transfection was carried out by following previously described procedures (Chen et al., 2006). After transfection, estradiol was applied to protoplasts immediately with final concentration of $20 \mu \mathrm{M}$. Protoplasts were harvested after incubation for 12, 24, 36 , and $48 \mathrm{~h}$, respectively, and subjected to GUS assay.

\section{Transformation of Rice Calli and Production of Transgenic Rice}

Plant expression constructs pUX-GUS, pXCL-GUS, pXCLFGUS, and pXCLF-PDSi were introduced into the Agrobacterium tumefaciens strain LBA4404. Rice callus was induced from the embryos of mature seeds of cv Nipponbare. Agrobacteriummediated transformation of rice calli was conducted using the method described previously (Hiei et al., 1994). The hygromycinresistant transgenic calli were subjected to estradiol induction assays or transferred to regeneration medium. Regenerated transgenic plants and their progeny were grown in a greenhouse. Transgenic lines contain a single copy of T-DNA insertion based on genetic segregation analysis of $\mathrm{T}_{1}$ generation, Southern blotting, and quantitative real-time PCR (qPCR) analysis of transgene copy number were used for further analyses (Supplementary Figure S1).

\section{Estradiol Treatment}

$17-\beta$-estradiol (Sigma-Aldrich, St. Louis, MO, United States) was dissolved to $20 \mathrm{mM}$ in dimethyl sulfoxide (DMSO), and stored at $-20^{\circ} \mathrm{C}$ as stock solution. The stock solution was added to sterilized water, or solid medium at a final concentration of $20 \mu \mathrm{M}$ immediately before use. The same volume of DMSO was added to the negative control solution or medium without estradiol. For induction treatment, rice transgenic calli were directly cultured on N6 medium supplemented with estradiol, and rice detached leaf pieces or rice seeds were submerged in estradiol water solution. In whole-plantlet treatment, seedlings germinated on $1 / 2 \mathrm{MS}$ medium plates were transferred to small tubes or flasks, and plantlet roots were submerged in estradiol water solution.

\section{GUS Assays}

GUS assays were performed as described by Jefferson et al. (1987). The fluorometric assay was carried out using 4methylumbelliferyl- $\beta$-D-glucuronide (MUG; Sigma-Aldrich, St. Louis, MO, United States) as substrate, and the histochemical staining was performed using X-Gluc (Sigma-Aldrich, St. Louis, MO, United States) as substrate.

\section{PCR, DNA Sequencing, qPCR, and qRT-PCR Analyses}

Genomic DNA was extracted from rice tissues by using the CTAB method as described by Murray and Thompson (1980). PCR for investigating the recombination events in induced rice plants was carried out under standard conditions. The PCR 


\section{pUX-GUS}

pXCL-GUS
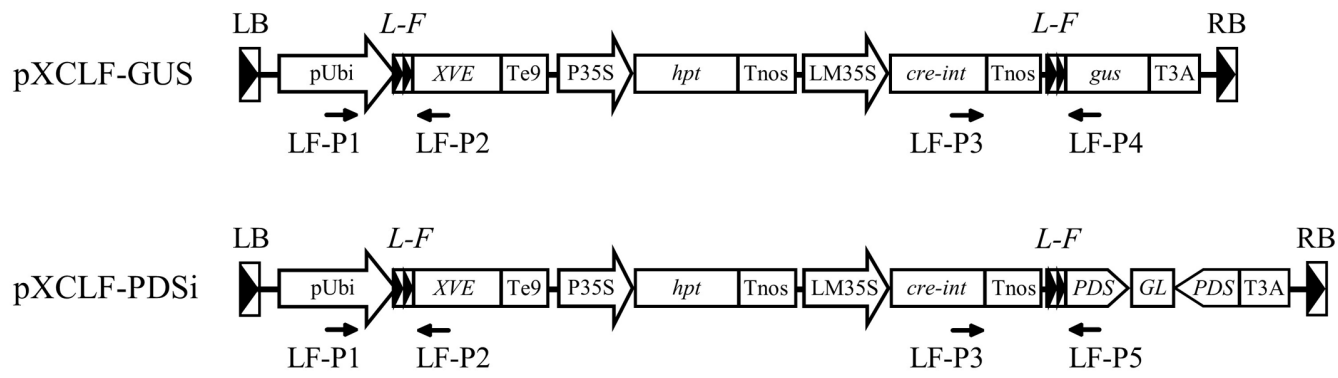

FIGURE 1 | Schematic diagrams of the T-DNA region of vectors used in this study. pUbi, maize ubiquitin-1 promoter; XVE, chimeric transactivator containing the regulator domain of an estrogen receptor (Zuo et al., 2000); Te9, rbcS E9 terminator; Pnos, nopaline synthase promoter; hpt, hygromycin phosphotransferase gene; Tnos, nopaline synthase terminator; LM35S, $8 \times$ LexA DNA binding site fused with the -46 CaMV 35S minimal promoter; gus, $\beta$-glucuronidase gene; cre-int, bacteriophage P1 Cre recombinase gene modified with an intron; T3A, rbcS 3A terminator; P35S, CaMV 35S promoter; PDS, a fragment of the rice phytoene desaturase gene; GL, gus linker fragment; loxP, specific recognition site of Cre; $L-F$, fusion sequence of loxP and FRT; LB, T-DNA left border; RB, T-DNA right border; LF-P1-LF-P5, primers used for PCR analysis.

products from primers LF-P1/LF-P4 or LF-P1/LF-P5 amplified from the induced LF-GUS or LF-PDSi plants were sequenced. The sequencing results were analyzed using Sequence Scanner software (Applied Biosystems). Real-time qPCR was performed to determine transgene copy number in homozygous lines by following previously described method (Wang et al., 2015). The single-copy rice endogenous gene RBE4 (Wang et al., 2015) was used as a reference and the Hpt gene was used as probe.

Total RNA was isolated from rice leaf tissues by using TRIzol reagent (Invitrogen Life Technologies, Carlsbad, CA, United States) and was subsequently treated with RNase-free DNase I (Takara, Dalian, China) to remove DNA contamination. Reverse transcription was carried out using $2.5 \mu \mathrm{g}$ of DNase I-treated total RNA with the RevertAid First Strand cDNA Synthesis Kit (Thermo Scientific, Lithuania, EU) according to the manufacturer's instructions. The qPCR was performed on an ABI 7500 real-time PCR system (Applied Biosystems, Carlsbad, CA, United States) using SYBR Premix Ex Taq (Takara, Dalian, China) according to the manufacturer's instructions.

All primers used for PCR, qPCR, and qRT-PCR were listed in the Supplementary Table S2.

\section{Southern Blot Analysis}

Southern blot analysis was performed following standard procedures (Sambrook and Russell, 2001). Rice genomic DNA (about $10 \mu \mathrm{g}$ ) was digested with appropriate restriction enzymes. The digested DNA was separated on a $0.8 \%$ agarose gel, and was transferred onto a Hybond-N+ nylon membrane (Amersham Biosciences, Buckinghamshire, United Kingdom). The blot was hybridized using a $3^{\prime}$-end fragment of the maize ubiquitin-1 promoter labeled by Alkphos Direct Labelling Reagents (Amersham Bioscience).

\section{RESULTS}

\section{XVE Stringently and Efficiently Regulates Gene Expression in Rice Cells and Tissues upon Direct Contact with \\ Estradiol}

We generated the estrogen-inducible expression construct $p U X$ GUS (Figure 1) using a gus gene as a reporter, because the GUS system has been proved to be a reliable and extremely sensitive reporter allowing both quantitative and histochemical assessment of gene activity in plants (Jefferson et al., 1987). The pUX-GUS construct was first tested transiently in rice protoplasts. The transfected rice protoplasts were treated with $20 \mu \mathrm{M}$ estradiol. Transient expression assays showed that the GUS expression level in transfected rice protoplasts was significantly induced after $12 \mathrm{~h}$ induction, and the induced level was increased gradually after 24, 36, and $48 \mathrm{~h}$ inductions (Figure 2A). In transfected rice protoplasts without treatment with estradiol, GUS expression was detected at a very low level after $24 \mathrm{~h}$ incubation, and remained at a similar low level after 36 or $48 \mathrm{~h}$ incubation (Figure 2A).

To further analyze XVE-controlled gene expression in stably integrated transgenic rice tissues, pUX-GUS and a control construct pCAMBIA1305.2 $2^{1}$ in which a gus gene is driven by a constitutive CaMV $35 \mathrm{~S}$ promoter (Odell et al.,

\footnotetext{
${ }^{1}$ http://www.cambia.org/daisy/cambia/585.html
} 


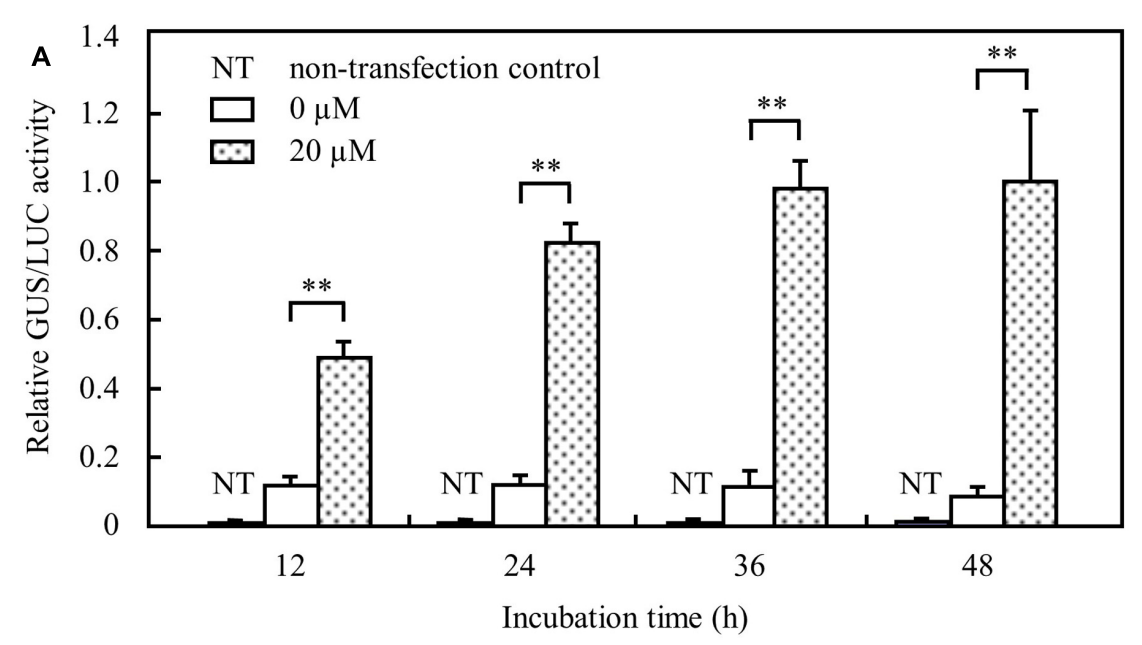

B

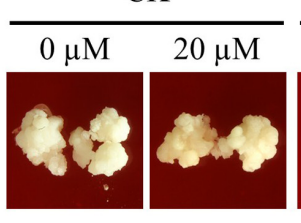

35S-GUS-14

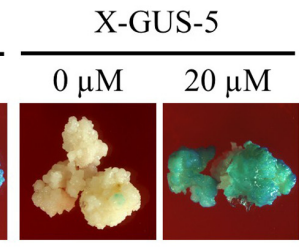

X-GUS-11
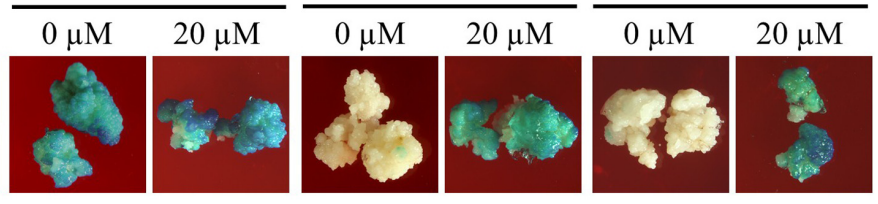

C

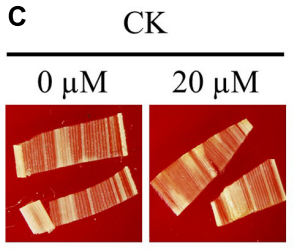

\begin{tabular}{|c|c|}
\hline $0 \mu \mathrm{M}$ & $20 \mu \mathrm{M}$ \\
\hline & 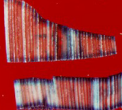 \\
\hline
\end{tabular}

\begin{tabular}{|c|c|}
\hline & \\
\hline $0 \mu \mathrm{M}$ & $20 \mu \mathrm{M}$ \\
\hline 11 & 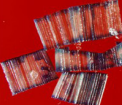 \\
\hline
\end{tabular}

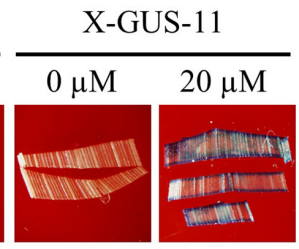

FIGURE 2 | XVE-controlled GUS expression in rice protoplast, calli, and leaf tissues upon direct contact with estradiol. (A) Transient expression assay of XVE-controlled GUS expression in rice protoplasts. Protoplasts transfected with construct pUX-GUS were treated with 0 or $20 \mu \mathrm{M}$ estradiol for $12,24,36$, and $48 \mathrm{~h}$, respectively. GUS expressions are represented as relative GUS/LUC activity. ${ }^{* *} P<0.01, t$-test. (B) GUS staining of rice calli induced with 0 or $20 \mu M$ estradiol. CK, non-transgenic wild type control; 35S-GUS-14, line \#14 of rice calli transformed with construct PCAMBIA1305, in which the gus gene was driven by a $35 S$ promoter; X-GUS-5 and X-GUS-11, line \#5 and \#11 of rice calli transformed with construct pUX-GUS. (C) GUS staining of detached transgenic leaf pieces induced with 0 or $20 \mu \mathrm{M}$ estradiol.

1985), were then used for stable transformation of rice calli. Hygromycin-resistant transgenic calli after selective screening were divided into two groups and were cultured on N6 medium with or without estradiol for induction analysis. Histochemical staining detected strong GUS expression in pUXGUS-transformed calli (named X-GUS) induced with $20 \mu \mathrm{M}$ estradiol for 3 days, similar to that of calli transformed with pCAMBIA1305.2 (named 35S-GUS) (Figure 2B). By contrast, almost no GUS staining was observed in X-GUS calli without treatment with estradiol during the culture period (Figure 2B).
Transgenic calli were further cultured to regenerate rice plants, and a total of 17 independent $\mathrm{T}_{0}$ X-GUS lines were obtained. Small pieces of transgenic plant leaves were cut and exposed to $20 \mu \mathrm{M}$ estradiol for $24 \mathrm{~h}$, and the GUS activity was determined by histochemical staining. From 17 independent lines, two lines showed no GUS staining in detached leaf pieces either treated or not treated with estradiol, and one line showed weak GUS staining in both induced and uninduced leaf pieces. For the remaining 14 lines, while no GUS staining was detected in leaf pieces without treatment, clear GUS staining was observed in estradiol treated-leaf pieces (Figure 2C). Taken together, these 
A

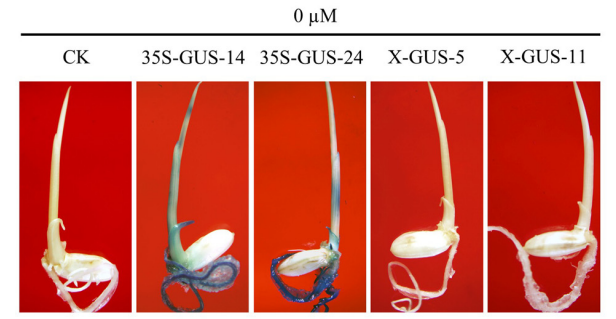

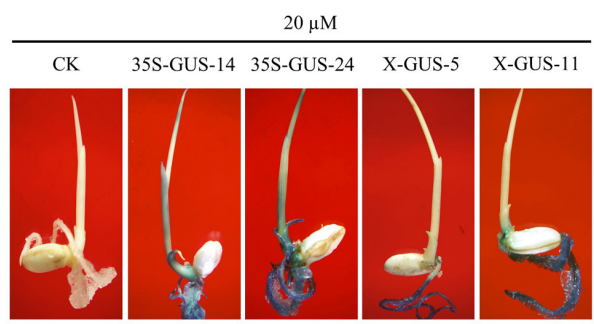

B

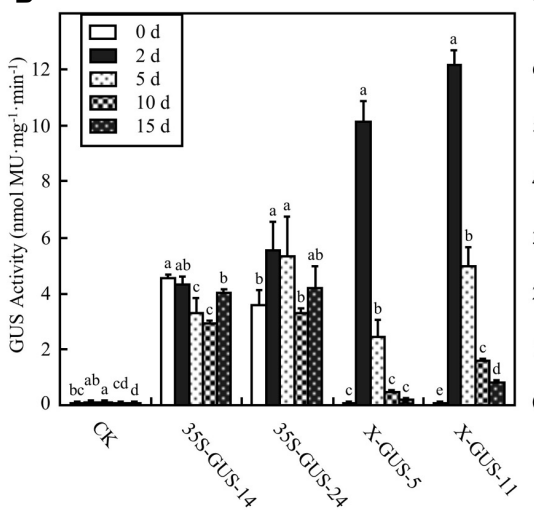

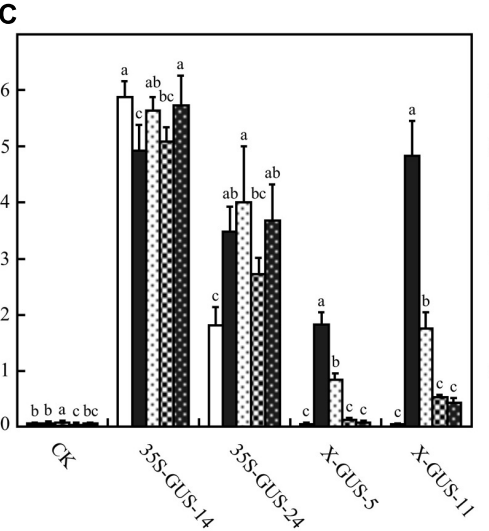

D

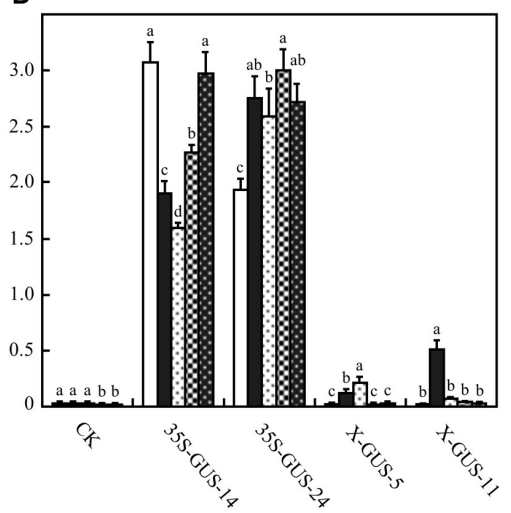

FIGURE 3 |XVE-controlled GUS expression in intact transgenic rice seedlings treated with estradiol through root absorption. (A) GUS staining of rice plantlets exposed to 0 or $20 \mu \mathrm{M}$ estradiol via root immersion for 2 days. CK, non-transgenic wild type control; 35S-GUS-14 and 35S-GUS-24, pCAMBIA1305-transformed rice line \#14 and \#24; X-GUS-5 and X-GUS-11, pUX-GUS-transformed rice line \#5 and \#11. (B-D) GUS fluorometric assay of the roots (B), stems (C), and leaves (D) of rice plantlets cultured in 0 or $20 \mu \mathrm{M}$ estradiol via root immersion. Three-leaf-old seedlings of rice plants were cultured in liquid solution, and the roots, shoots, or leaves of the cultured seedlings were collected for GUS assay on days 0, 2, 5, 10, and 15, respectively. Different letters indicate groups with significant differences (ANOVA, $p<0.05)$.

results demonstrated that the XVE system could stringently and efficiently regulate gene expression in rice cells and tissues.

\section{XVE Mediates Low Level Inducibility of Target Gene in the Leaves of Intact Plants Induced with Estradiol through Root Absorption}

The transgenic X-GUS, and 35S-GUS rice plants were grown to produce progeny of advanced generation for further analysis. Among the 14 screened X-GUS lines, \#5 and \#11 were examined to confer a strong estradiol-inducible GUS expression pattern in detached leaf pieces, and contain a single copy of T-DNA insertion based on genetic segregation analysis of $\mathrm{T}_{1}$ generation, Southern blotting and qPCR analysis of transgene copy number (Supplementary Figure S1). Detached leaf pieces of X-GUS plants of each different generation $\left(\mathrm{T}_{1}\right.$ to $\left.\mathrm{T}_{4}\right)$ were treated with estradiol, and GUS staining could be observed in induced leaf tissues but not in uninduced tissues, similar to that of $\mathrm{T}_{0}$ generation plants (Figure 2C). These results suggested that XVE-controlled GUS expression pattern could be inherited stably over generations.

To examine the estrogen-induced target gene expression patterns in intact rice plantlets, 4-day-old seedlings of $\mathrm{T}_{5}$ homozygous lines were cultured in liquid solution via a root absorption manner for 2 days. The cultured seedlings were subjected to histochemical staining. While no GUS expression was observed in plantlets of X-GUS-\#5 or X-GUS-\#11 cultured in liquid medium without estradiol, strong GUS staining was observed in the roots of X-GUS-\#5 and X-GUS-\#11 cultured in estradiol solution (Figure 3A). However, when compared with 35S-GUS plants, X-GUS plantlets showed only weak GUS staining in leaf tissues (Figure 3A). A fluorometric assay was also performed to detect GUS expression level in transgenic plantlets. Three-leaf-stage seedlings of transgenic plants were cultured in liquid solution for different induction periods $(0$, $2,5,10$, and 15 days), and the roots, shoots, and leaves of the cultured seedlings were collected and evaluated for GUS activity. With $20 \mu \mathrm{M}$ estradiol induction for 2 days, GUS expression was induced in X-GUS plantlets. In the roots of X-GUS-\#5 and X-GUS-\#11, GUS level was about two times higher than or similar to that of 35S-GUS plants (Figures 3B,C). However, GUS level in the leaves of X-GUS\#5 and X-GUS-\#11 was much lower than that of 35S-GUS plants (Figure 3D), similar to previous report that XVEcontrolled GFP was expressed at a low level in the leaves of transgenic seedlings cultured on the inductive medium (Okuzaki et al., 2011). Fluorometric assays also revealed that GUS expression level in X-GUS plantlets was gradually decreased after induction for 5, 10, and 15 days, respectively (Figures 3B-D). 
A

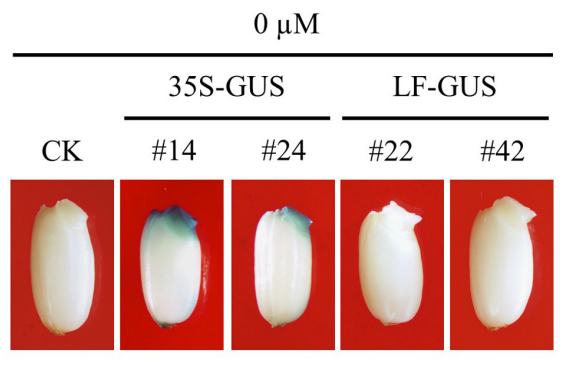

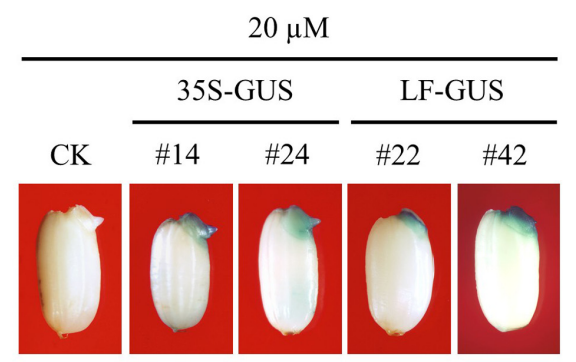

8

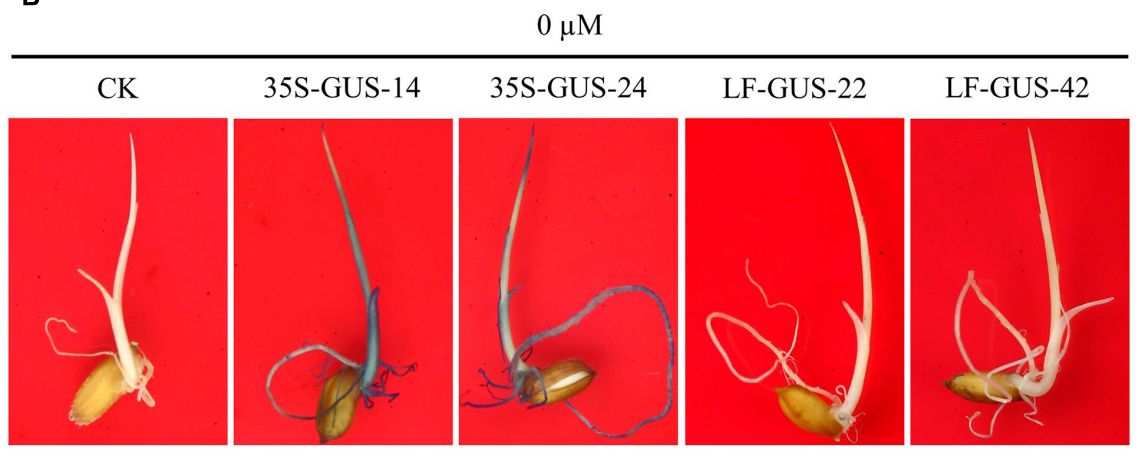

$20 \mu \mathrm{M}$

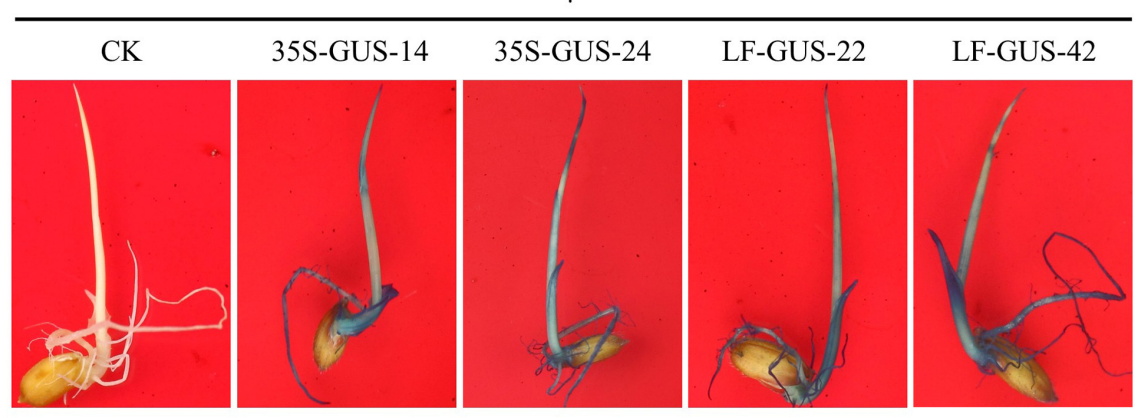

FIGURE 4 | Induced GUS expression in germinating embryos and seedlings of LF-GUS transgenic rice. (A) GUS staining of rice seeds soaked in 0 or 20 $\mu$ M estradiol for 3 days. CK, non-transgenic wild type control; 35S-GUS-14 and 35S-GUS-24, pCAMBIA1305-transformed rice line \#14 and \#24; LF-GUS-22 and LF-GUS-42, pXCLF-GUS-transformed rice line \#22 and \#42. (B) GUS staining of seedlings developed from seeds soaked and germinated in liquid solution with 0 or $20 \mu \mathrm{M}$ estradiol.

\section{Construction of an Improved Chemical-Regulated, Site-Specific Recombination System for Rice}

Unlike Arabidopsis, rice plants are larger in size, and have a relatively longer life cycle. The difficulty in inducing target genes to be expressed at a high level in the leaves of intact plantlets, and the relatively short induction duration, have limited the use of the XVE system in rice. We reasoned that a system permitting constitutive gene expression after chemical induction would be more feasible for rice. We thus designed an alternative approach by employing the XVE system coupled with a sitespecific recombination cassette. A basic construct pXCL-GUS, in which a XVE-controlled Cre/loxP-mediated recombination would lead to fusion of a maize (Zea mays) ubiquitin-1 promoter (Christensen et al., 1992) to the gus gene upon estradiol induction (Figures 1, 6D), was developed initially. However, rice transformation experiments showed that pXCL-GUS gave only very low transformation efficiency (Supplementary Table S3). A modified construct pXCLF-GUS (Figure 1), in which the original nos promoter for the selectable marker gene $h p t$ was replaced with a CaMV 35 S promoter, was constructed. In pXCLFGUS, the two directly oriented loxP sequences were also replaced with two loxP-FRT fusion sequences which were previously reported to have enhanced excision efficiency mediated by Cre recombinase (Luo et al., 2007). Rice transformation experiments 


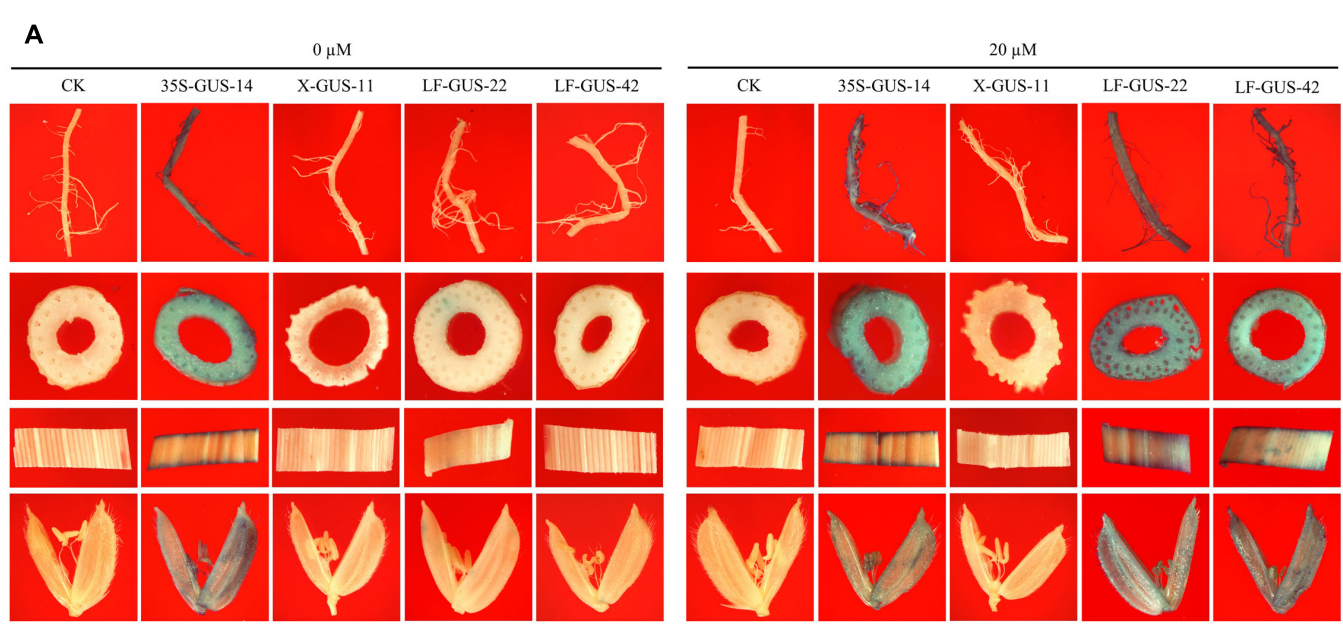

B

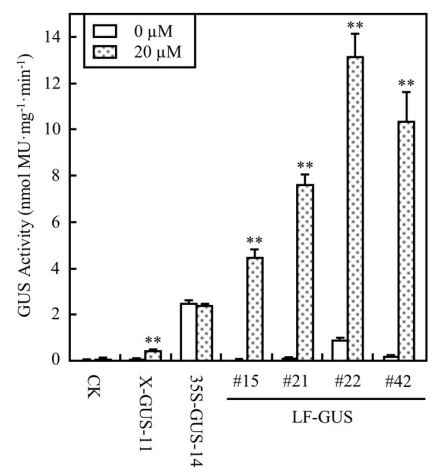

C

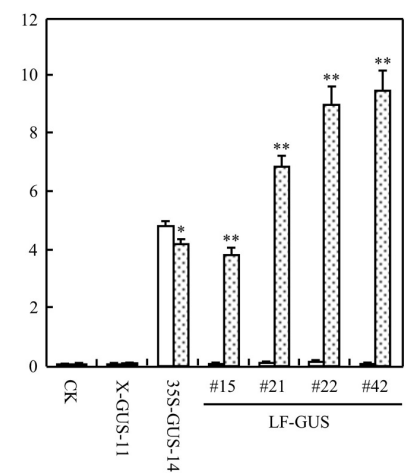

D

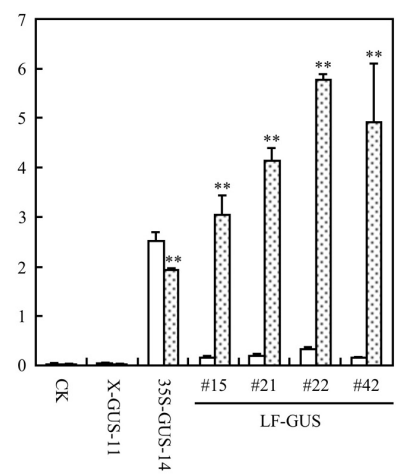

FIGURE 5 | Induced GUS expression in mature LF-GUS transgenic rice plants. (A) GUS staining of the roots, stems, leaves, and florets of mature plants developed from seeds soaked in liquid solution with 0 or $20 \mu \mathrm{M}$ estradiol. CK, non-transgenic wild type control; 35S-GUS-14, pCAMBIA1305-transformed rice line \#14; X-GUS-11, pUX-GUS-transformed rice line \#11; LF-GUS-22 and LF-GUS-42, pXCLF-GUS-transformed rice line \#22 and \#42. (B-D) GUS fluorometric assay of the roots (B), stems (C) and leaves (D) of mature rice plants developed from seeds soaked and germinated in liquid solution with 0 or $20 \mu \mathrm{M}$ estradiol. CK, non-transgenic wild type control; X-GUS-11, pUX-GUS-transformed rice line \#11; 35S-GUS-14, pCAMBIA1305-transformed rice line \#14; LF-GUS \#15, \#21, \#22, and \#42, pXCLF-GUS-transformed rice line \#15, \#21, \#22, and \#42. Statistical significances $\left({ }^{*} P<0.05\right.$ or $\left.* * P<0.01\right)$ between plants developed from seeds soaked in 0 or $20 \mu \mathrm{M}$ estradiol were analyzed by Student's $t$-test.

showed that the modified pXCLF-GUS yielded a transformation efficiency about 15 times higher than that of pXCL-GUS (Supplementary Table S3).

\section{The XVE-controlled Cre/loxP-FRT (XCLF) System Permits Inducible and High-Level Expression of Target Genes in Transgenic Rice Plants}

The uninduced rice calli transformed with pXCLF-GUS (named LF-GUS) were cultured on media for regeneration, and a total of 18 independent $\mathrm{T}_{0}$ plants were obtained. $\mathrm{T}_{0}$ plants were transferred to soil and maintained in a greenhouse to produce $\mathrm{T}_{1}$ seeds. To induce expression of Cre recombinase, we soaked transgenic rice seeds in liquid solution instead of germinating seeds on solid medium. A 3-day soaking of seeds was observed to have a good effect on seed induction (Figure 4A). By histochemical staining assay, about $12 \mathrm{~T}_{1}$ independent lines were detected to display induced GUS expression upon seed-soaking treatment. In LF-GUS lines, GUS expression was detected in the embryos of LF-GUS transgenic seeds soaked in the inductive solution, but not in seeds soaked in the solution without estradiol (Figure 4A). The LF-GUS seedlings developed from induced seeds displayed a constitutive expression pattern of GUS, whereas no GUS or only very faint GUS spots were observed in uninduced LF-GUS seedlings (Figure 4B).

The $\mathrm{T}_{2}$ generation of four LF-GUS homozygous lines, \#15, $\# 21$, \#22, and \#42 were subjected to more detailed analysis. In mature flowering plants developed from estradiol-induced LFGUS seeds, constitutive GUS expression was detected in root, stem, leaf, and floret tissues of LF-GUS plants by histochemical staining (Figure 5A). Consistent with results observed in $T_{1}$ seedlings, almost no GUS staining or only very faint GUS spots were observed in tissues of LF-GUS plants developed from uninduced seeds (Figure 5A). Fluorometric assays confirmed that GUS was highly expressed in all tested root, stem, and leaf 

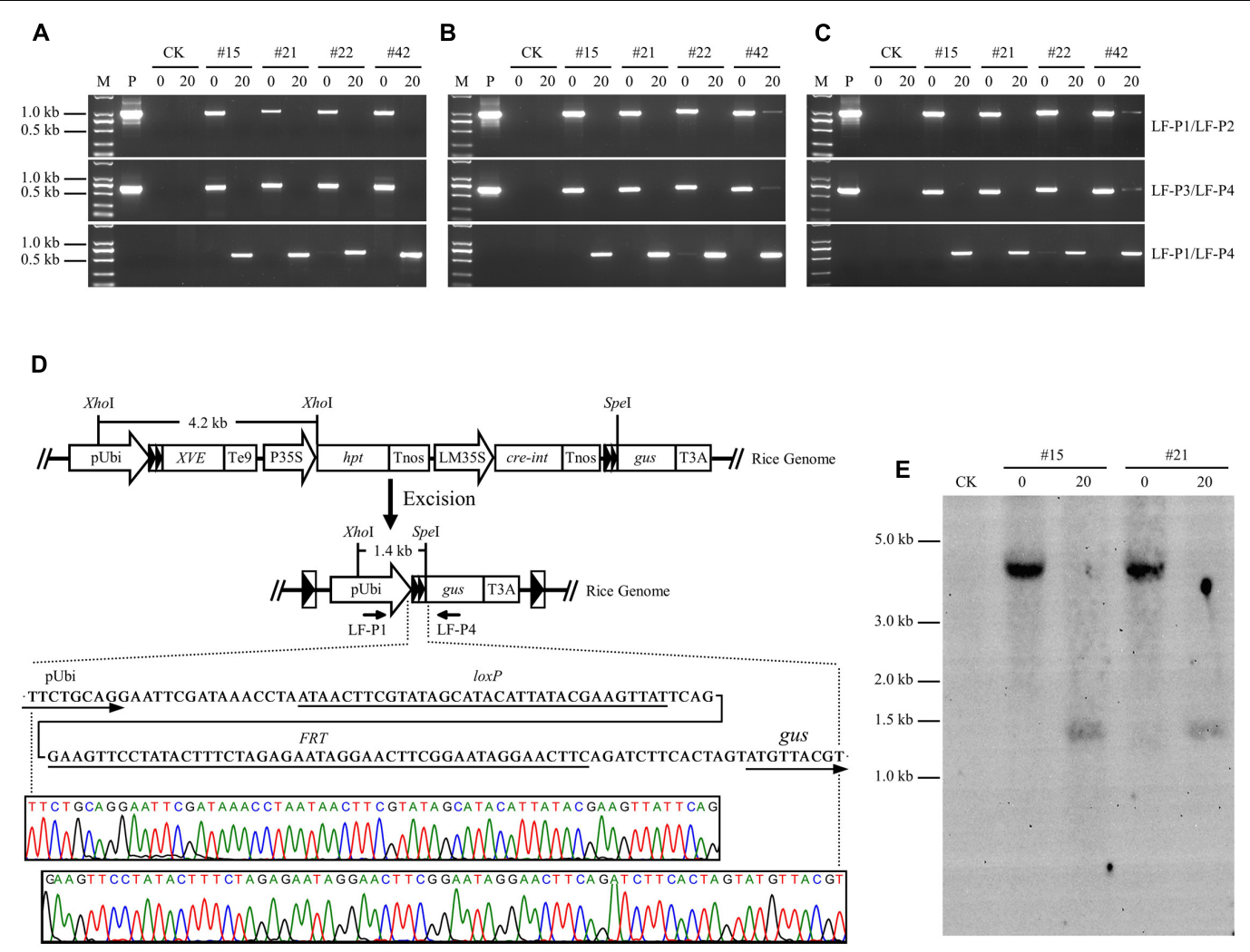

FIGURE 6 | Molecular characterization of chemical-induced site-specific DNA recombination in LF-GUS transgenic rice plants. (A-C) PCR analysis of genomic DNA extracted from the roots (A), stems (B), and leaves (C) of LF-GUS plants. M, DNA molecular marker; P, plasmid control pXCLF-GUS; CK, non-transgenic wild type control; \#15, \#21, \#22, and \#42, pXCLF-GUS-transformed rice line \#15, \#21, \#22, and \#42; 0, plants developed from seeds soaked and germinated in liquid solution with $0 \mu \mathrm{M}$ estradiol; 20, plants developed from seeds soaked and germinated in liquid solution with $20 \mu \mathrm{M}$ estradiol. (D) Sequence confirmation of site-specific DNA recombination in the genome of induced LF-GUS plants. The diagram represents the putative reconstituted structure after recombination between two loxP-FRT sites. Sequence below the diagram indicates the predicted nucleotide sequence of the junction regions at the recombined loxP-FRT site. The bottom chromatogram shows sequencing results of the LF-P1/LF-P2 amplified product from induced LF-GUS plants. (E) Southern blot analysis of genomic DNA extracted from leaves of LF-GUS plants. CK, non-transgenic wild type control; \#15 and \#21, pXCLF-GUS-transformed rice line \#15 and \#21; 0, plants developed from seeds soaked and germinated in liquid solution with $0 \mu \mathrm{M}$ estradiol; 20, plants developed from seeds soaked and germinated in liquid solution with $20 \mu \mathrm{M}$ estradiol. Rice genomic DNA was digested with Xhol and Spel. The blot was hybridized with a $3^{\prime}$-end fragment of the maize ubiquitin-1 promoter.

tissues in the induced mature LF-GUS plants (Figures 5B-D). While the level of GUS activity in root, stem, or leaf tissues of LF-GUS-\#15 plants were comparable to that of 35S-GUS-\#14 plants, GUS expression level of LF-GUS-\#21, \#22, or \#42 plants was about 1.5-2 times higher than that of 35S-GUS\#14 plants (Figures 5B-D). In uninduced LF-GUS plants, only a low GUS level similar to that of non-transgenic plants was detected (Figures 5B-D). No GUS expression was detected in tissues of mature plants of X-GUS line \#11 developed from seeds germinated in liquid solution with or without inducer (Figures 5A-D), indicating that estradiol induction in seeds did not persist to later times.

PCR analysis was performed on genomic DNA isolated from root, stem, and leaf tissues to investigate the recombination events in LF-GUS plants. Using specific primers LF-P1/LF$\mathrm{P} 2$ targeted to the joint region of $p U b i-l o x P-F R T-X V E$, and LF-P3/LF-P4 to the joint region of Cre-int-Tnos-loxP-FRTgus of the non-recombinant T-DNA (Figure 1), the expected 1019 and 679 bp fragments were detected in the tissues of uninduced LF-GUS plants, respectively (Figures 6A-C). No LF-P1/LF-P2 or LF-P3/LF-P4 fragments were detected in the tissues of induced LF-GUS plants except for line \#42 showing a faint band, indicating highly efficient DNA recombination in induced plants. By contrast, PCR amplifications using primers LF-P1/LF-P4 generated a 626 bp fragment consistent with reconstitution of the $p U b i-l o x P-F R T$-gus transcription unit (Figure 6D) in induced LF-GUS plants, except that a faint band was detected in uninduced line \# 22 (Figures 6A-D), indicating no DNA or almost no DNA recombination in uninduced plants. The PCR products of LF-P1/LF-P4 fragments amplified from induced plants of the four LF-GUS plants were sequenced. And sequencing results from all four lines were identical, showing that the maize ubiquitin-1 promoter was contiguous with one copy of loxP-FRT sequence, followed by the gus sequence (Figure 6D). DNA isolated from LF-GUS-\#15 and LF-GUS-\#21 plants was double cut with XhoI and SpeI (Figure 6D), and Southern blot analysis was performed using a $3^{\prime}$-end fragment of the maize ubiquitin-1 promoter as a probe. As showed in Figure 6E, an 


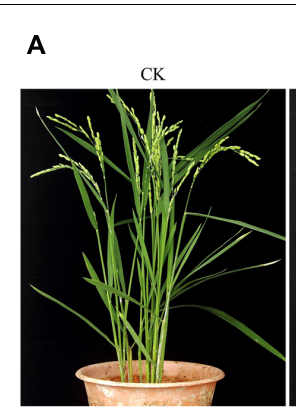

C

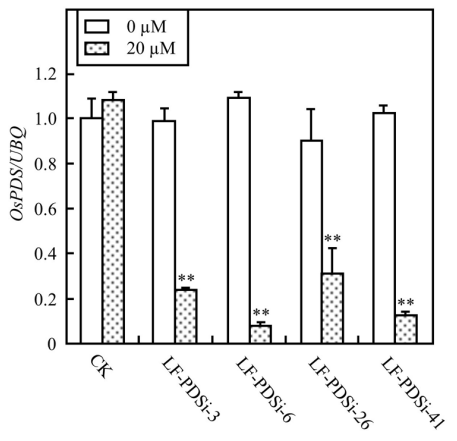

LF-PDSi-26
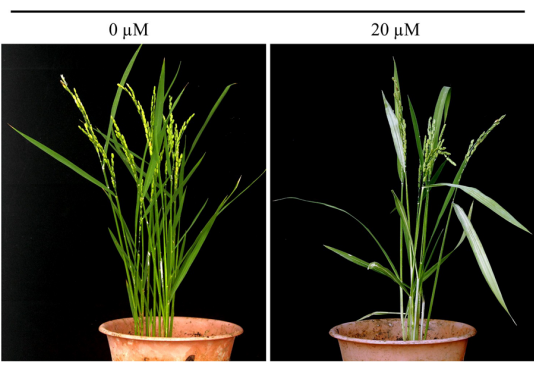

B

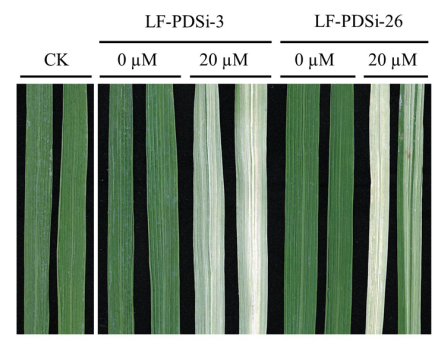

D

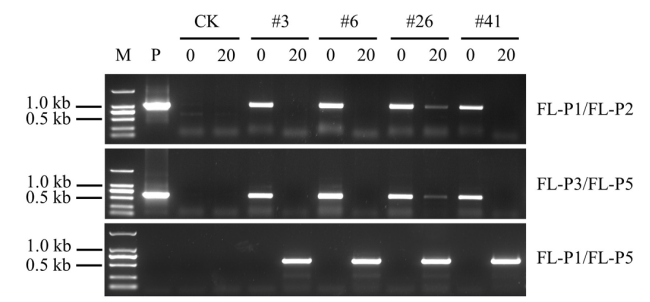

FIGURE 7 | Induced OSPDS silencing in mature LF-PDSi transgenic rice plants. (A) Phenotype of mature plants developed from seeds soaked in liquid solution with 0 or $20 \mu \mathrm{M}$ estradiol. CK, non-transgenic wild type control; LF-PDSi-26, pXCLF-PDSi-transformed rice line \#26. (B) Phenotype of leaves from mature plants developed from seeds soaked in liquid solution with 0 or $20 \mu \mathrm{M}$ estradiol. CK, non-transgenic wild type control; LF-PDSi-3, LF-PDSi-26, pXCLF-PDSi-transformed rice line \#3 and \#26. (C) qRT-PCR analysis of endogenous OsPDS mRNA level in mature plants developed from seeds soaked in liquid solution 0 or $20 \mu \mathrm{M}$ estradiol. CK, non-transgenic wild type control; LF-PDSi-3, LF-PDSi-6, LF-PDSi-26, LF-PDSi-41, pXCLF-PDSi-transformed rice line \#3, \#6, \#26, and \#41. Statistical significances $\left({ }^{* *} P<0.01\right)$ between plants developed from seeds soaked in 0 or in $20 \mu \mathrm{M}$ estradiol were analyzed by Student's $t$-test. (D) PCR analysis of chemical-induced site-specific DNA recombination in LF-PDSi transgenic rice plants. P, plasmid control pXCLF-PDSi; CK, non-transgenic wild type control; \#3, \#6, \#26, and \#41, pXCLF-PDSi-transformed rice line \#3, \#6, \#26, and \#41; 0, plants developed from seeds soaked and germinated in liquid solution with $0 \mu \mathrm{M}$ estradiol; 20, plants developed from seeds soaked and germinated in liquid solution with $20 \mu \mathrm{M}$ estradiol.

expected $4.2 \mathrm{~kb}$ band was detected in uninduced LF-GUS-\#15 and LF-GUS-\#21. By contrast, a $1.4-\mathrm{kb}$ band was detected in induced LF-GUS-\#15 and LF-GUS-\#21 plants, confirming the recombination events in LF-GUS plants.

\section{Inducible Silencing of Endogenous OsPDS in Transgenic Rice Plants}

In addition to ectopic expression of target genes, the XCLF system was also tested for gene knock-down in rice plants. A RNAi construct pXCLF-PDSi (Figure 1), which contained an hpRNAi cassette targeted to a rice phytoene desaturase (OsPDS) gene, was developed and introduced into rice by Agrobacterium-mediated transformation. A total of 14 independent $\mathrm{T}_{0}$ plants (named LF-PDSi) were obtained, and no uninduced plants showed detectable albino phenotype. In the $\mathrm{T}_{1}$ generation, eight lines of induced transgenic plants showed a clear albino phenotype (Figures 7A,B), whereas all transgenic lines developed from seeds geminated in liquid solution without estradiol grew similar to non-transgenic control plants. Real-time RT-PCR analysis showed that the endogenous OsPDS mRNA level was greatly reduced in the induced plants (Figure $7 \mathrm{C}$ ). Using the same primers LF-P1, LF-P2, and LF-P3 used for detecting LF-GUS plants, and a specific primer LF-P5 targeted to the hpRNAi cassette of the OsPDS fragment (Figure 1), PCR analysis detected LF-P1/LF-P2, LF-P3/LF-P5 non-recombinant fragments only in uninduced plants, except for induced line \#26 showing faint amplified bands (Figure 7D). By contrast, PCR analysis detected a 583 bp LF-P1/LF-P5 recombinant fragment only in induced LF-PDSi plants (Figure 7D). Moreover, sequencing results of the LF-P1/LF-P5 amplified products from induced LF-PDSi plants indicated the accurate reconstitution of the recombinant cassette of $p U b i$-loxP-FRT-hpRNAi (OsPDS) (Supplementary Figure S2). These results confirmed that the albino phenotype in induced LF-PDSi plants resulted from knock-down of OsPDS gene expression mediated by chemically regulated recombination of the XCLF system.

\section{DISCUSSION}

In previous studies, gene induction systems such as the GVG system (Ouwerkerk et al., 2001; Yoshida et al., 2009), the XVE system (Sreekala et al., 2005; Okuzaki et al., 2011), and the GAL4-ER-VP16 system (Zhang et al., 2007) have been applied to conditionally express target genes in rice. GVG consists of a DNA-binding domain of the yeast transcription factor GAL4, a VP16, and a rat glucocorticoid receptor (Aoyama and Chua, 


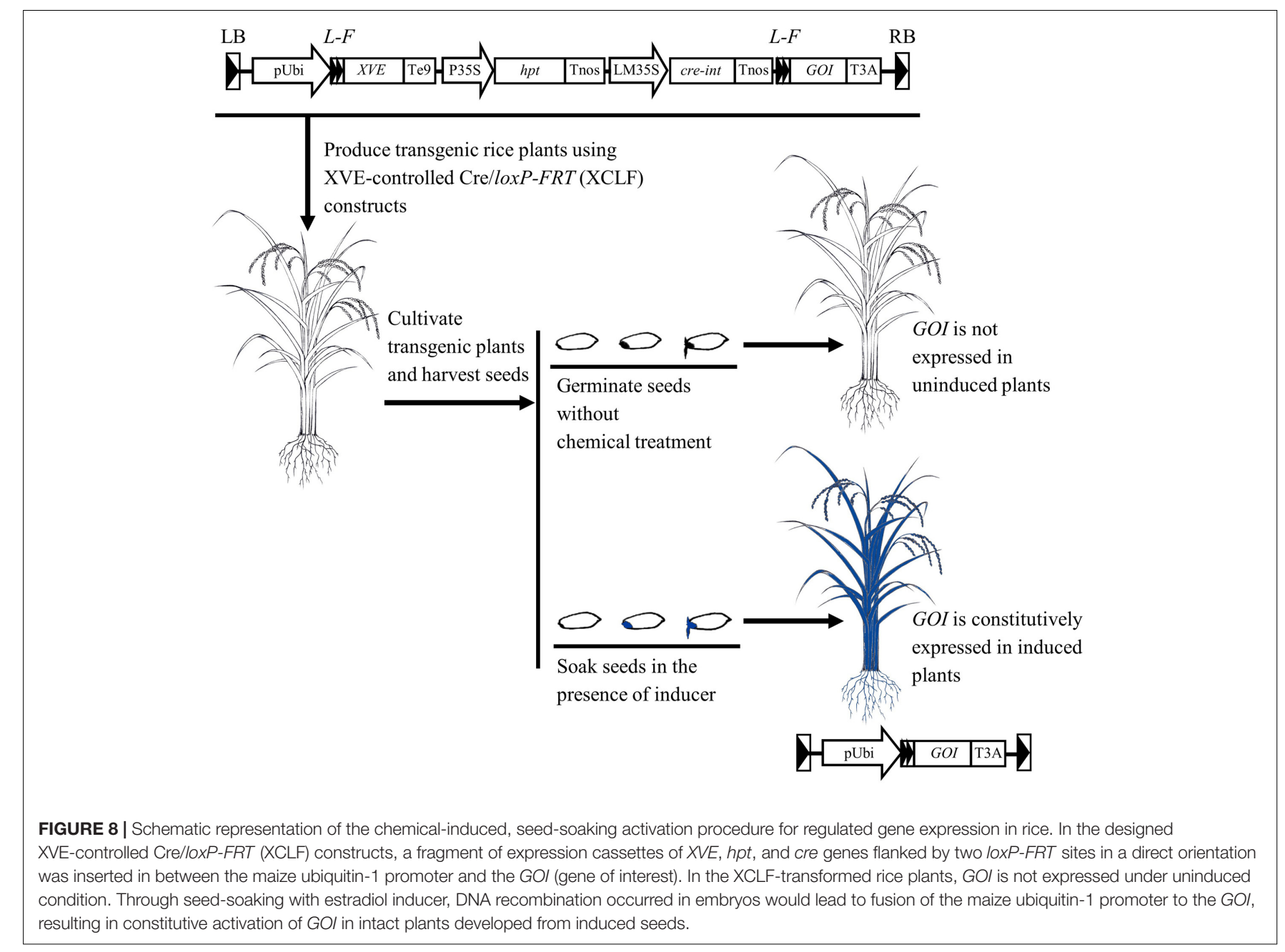

1997). The transcriptional activity of GVG is regulated by glucocorticoid dexamethasone, however, it has been found to cause growth defects in Arabidopsis (Kang et al., 1999), lotus (Andersen et al., 2003), tobacco (Amirsadeghi et al., 2007), as well as in rice (Ouwerkerk et al., 2001). The XVE and the GAL4-ER-VP16 systems were developed based on the human estrogen receptor binding domain, and both were regulated by estradiol. While it has not been determined whether the GAL4-ER-VP16 system has toxic or physiological effects on rice plants (Zhang et al., 2007), transgenic rice seedlings expressing a XVE-controlled GFP construct showed no morphological differences compared with non-transgenic control lines (Okuzaki et al., 2011). Similarly, in our study, we observed similar plant heights and seed setting rates between XVE transgenic lines and non-transgenic wild type plants. However, it remains to be investigated whether the XVE system could affect other important agronomic traits in rice.

By using the firefly luciferase system as a reporter, Zhang et al. (2007) demonstrated that the inducibility of target gene expression controlled by the GAL4-ER-VP16 system could reach up to 10,000-fold in rice tissues directly exposed to estradiol. In rice calli stably transformed with a XVE-controlled $g f p$ construct, while no GFP signal was detected in calli cultured in media without estradiol, GFP signals were detected in the entire surface of calli cultured in media containing $>5 \mu \mathrm{M}$ estradiol within 2 days (Okuzaki et al., 2011). Similarly, in our study, high inducibility of GUS was detected in pUXGUS-transfected rice protoplast, and in X-GUS calli or X-GUS leaf pieces upon estradiol induction. More importantly, no GUS or almost no GUS expression was observed in uninduced calli or leaf pieces (Figures 2B,C), confirming again that the XVE system is highly inducible and tightly regulated in rice cells. However, in intact plantlets induced with estradiol via root immersion, while the roots of the plantlets displayed high inducibility of GUS, the stems and the leaves expressed a much lower level of GUS. Okuzaki et al. (2011) discussed two possible reasons-inefficient estradiol uptake or low XVE expression level, for low level of target gene induction in the leaves of the intact plantlets. Unlike the previous study in which XVE expression was controlled by a weak G10-90 promoter (Okuzaki et al., 2011), in our study, XVE was driven by a maize ubiquitin-1 promoter (Figure 1), which gives constitutive and high-level expression of transgenes in rice (Christensen and Quail, 1996). In addition, in the present study, estradiol 
was applied at a high concentration of $20 \mu \mathrm{M}$, which was two times higher than the reported saturated concentration for induction (Okuzaki et al., 2011). The fact that GUS activities in the roots of estradiol-induced X-GUS seedlings were about two times higher than that of 35S-GUS plants (Figure 3B) also suggested that estradiol uptake and XVE level should be sufficient for activating target gene induction. Therefore, the most likely reason for low inducibility of target gene expression in the leaves of intact plantlets could be that systemic movement of estradiol was limited within the rice plant.

To meet the need for chemical-regulated, high-level expression of target gene in intact rice plants, we have designed an alternative approach by employing the XVE system coupled with a site-specific recombination cassette. In the backbone of the original XVE constructs pER8 (Zuo et al., 2000), the selectable $h p t$ gene for hygromycin resistance was controlled by a weak nopaline synthase promoter (Sanders et al., 1987). In the constructs pXCLF-GUS and pXCLF-PDSi, we replaced the nopaline synthase promoter with a strong CaMV 35S promoter (Figure 1). Transformation experiments indicated that this modification greatly enhanced the rice transformation efficiency of the constructs (Supplementary Table S3). By using a GUS reporter, we tested the feasibility of the XCLF system in rice. Histochemical staining of LF-GUS rice seeds soaked in estradiol solution for 3 days showed that GUS was expressed strongly in the germinating embryo (Figure 4A), indicating that uptake of estradiol by rice seeds at early stages of germination could efficiently induce Cre/loxP-FRT-mediated DNA excision in rice embryos. Consistent with this, rice seedlings and matured plants developed from induced seeds displayed a constitutive GUS expression pattern (Figures $\mathbf{4 B}, \mathbf{5 A} \mathbf{A}-\mathbf{D}$ ), and the GUS expression level in the roots, stems, or leaves were higher or at least similar to that of transgenic rice plants expressing GUS under control of a 35S promoter (Figures 5B-D). This property of the XCLF system would allow target genes to be expressed at the desired high level for functional characterization. In the present study, the XCLF system was also tested for knocking down target gene expression. We tested the XCLF system using an hpRNAi cassette for the OSPDS gene. The clear albino phenotype and the reduction of OsPDS mRNA of induced LF-PDSi plants indicated that the system could be applied in knocking down endogenous gene expression in rice with high efficiency.

In a previous report, Sreekala et al. (2005) observed that DNA recombination mediated by a XVE-controlled Cre/loxP system was not efficiently induced in the germline cells in rice. In the present study, we detected highly efficient induced DNA excision in germinating rice embryos. Molecular analyses of induced rice plants displaying strong recombinant phenotypes showed that complete or almost complete DNA excision occurred in the tested plants (Figures 6A-C, 7D). The possible reason for high efficiency of induced DNA recombination in our experiments could be the efficient uptake of estradiol in germinating embryos by the soaking treatment of rice seeds. Unlike previous work, where transgenic seeds were germinated on solid inductive media (Zuo et al., 2001; Guo et al., 2003; Sreekala et al.,
2005), we have optimized a seed-soaking protocol for XVE induction. Since uptake of water is the initial event for plant seed germination (Bewley, 1997), soaking seeds in estradiol solution would allow the inducer to access the embryos and induce Cre recombinase at a very earlier stage, resulting in highly efficient DNA recombination.

\section{CONCLUSION}

The chemical-induced, seed-soaking activation procedure based on the XCLF system appears to be effective in regulating gene expression in intact rice plants (Figure 8), therefore providing a useful tool for functional genomics and biotechnology applications such as the deletion of selectable marker genes in rice.

\section{DATA ACCESSIBILITY}

The sequence data from this article can be found in the GenBank database, under accession numbers MF434112 (pUXGUS), MF434111 (pXCL-GUS), MF434113 (pXCLF-GUS), and MF434110 (pXCLF-PDSi).

\section{AUTHOR CONTRIBUTIONS}

ZJC, G-LW, FW, and SC conceived and designed the experiments. ZJC, QC, CH, XG, ZQC, YL, TH, and $\mathrm{MB}$ performed the experiments. ZJC, QC, ZW, FW, and SC analyzed the data. GL and ZW contributed reagents and tools. ZJC and SC wrote the manuscript.

\section{FUNDING}

This research was supported by National Major Special Project on New Varieties Cultivation for Transgenic Organisms, China (2016ZX08001001-002), National Natural Science Foundation of China (U1405212, 31471760), and by the Science and Technology Plan of Fujian Province, China (2014R1019-3).

\section{ACKNOWLEDGMENTS}

We are grateful to Dr. Zhongchao Yin, Temasek Life Sciences Laboratory, National University of Singapore, Singapore, for kindly providing the pUH-GFP2 plasmid, and Dr. Daniel J. Ebbole for editing of the manuscript.

\section{SUPPLEMENTARY MATERIAL}

The Supplementary Material for this article can be found online at: http://journal.frontiersin.org/article/10.3389/fpls.2017.01447/ full\#supplementary-material 


\section{REFERENCES}

Amirsadeghi, S., McDonald, A. E., and Vanlerberghe, G. C. (2007). A glucocorticoid-inducible gene expression system can cause growth defects in tobacco. Planta 226, 453-463. doi: 10.1007/s00425-007-0495-1

Andersen, S. U., Cvitanich, C., Hougaard, B. K., Roussis, A., Grønlund, M., Jensen, D. B., et al. (2003). The glucocorticoid-inducible GVG system causes severe growth defects in both root and shoot of the model legume Lotus japonicus. Mol. Plant Microbe Interact. 16, 1069-1076. doi: 10.1094/MPMI.2003.16. 12.1069

Aoyama, T., and Chua, N. H. (1997). A glucocorticoid-mediated transcriptional induction system in transgenic plants. Plant J. 11, 605-612. doi: 10.1046/j.1365313X.1997.11030605.x

Bewley, J. D. (1997). Seed germination and dormancy. Plant Cell 9, 1055-1066. doi: 10.1105/tpc.9.7.1055

Bohner, S., Lenk, I., Rieping, M., Herold, M., and Gatz, C. (1999). Transcriptional activator TGV mediates dexamethasone-inducible and tetracyclineinactivable gene expression. Plant J. 19, 87-95. doi: 10.1046/j.1365-313X.1999. 00503.x

Bruce, W., Folkerts, O., Garnaat, C., Crasta, O., Roth, B., and Bowen, B. (2000). Expression profiling of the maize flavonoid pathway genes controlled by $\beta$-estradiol-inducible transcription factors CRC and P. Plant Cell 12, 65-80. doi: $10.1105 /$ tpc.12.1.65

Caddick, M. X., Greenland, A. J., Jepson, I., Krause, K. P., Qu, N., Riddell, K. V., et al. (1998). An ethanol inducible gene switch for plants used to manipulate carbon metabolism. Nat. Biotechnol. 16, 177-180. doi: 10.1038/nbt0298-177

Chen, S., Tao, L., Zeng, L., Vega-Sanchez, M. E., Umemura, K., and Wang, G. L. (2006). A highly efficient transient protoplast system for analyzing defence gene expression and protein-protein interactions in rice. Mol. Plant Pathol. 7, 417-427. doi: 10.1111/j.1364-3703.2006.00346.x

Christensen, A. H., and Quail, P. H. (1996). Ubiquitin promoter-based vectors for high level expression of selectable and/or screenable marker genes in monocotyledonous plants. Transgenic Res. 5, 213-218. doi: 10.1007/ BF01969712

Christensen, A. H., Sharrock, R. A., and Quail, P. H. (1992). Maize polyubiquitin genes: structure, thermal perturbation of expression and transcript splicing, and promoter activity following transfer to protoplasts by electroporation. Plant Mol. Biol. 18, 675-689. doi: 10.1007/BF00020010

De Veylder, L., Van Montagu, M., and Inze, D. (1997). Herbicide safener-inducible gene expression in Arabidopsis thaliana. Plant Cell Physiol. 38, 568-577. doi: 10.1093/oxfordjournals.pcp.a029206

Guo, H. S., Fei, J. F., Xie, Q., and Chua, N. H. (2003). A chemical-regulated inducible RNAi system in plants. Plant J. 34, 383-392. doi: 10.1046/j.1365313X.2003.01723.x

Hiei, Y., Ohta, S., Komari, T., and Kumashiro, T. (1994). Efficient transformation of rice (Oryza sativa L.) mediated by Agrobacterium and sequence analysis of the boundaries of the T-DNA. Plant J. 6, 271-282. doi: 10.1046/j.1365-313X. 1994.6020271.x

International Rice Genome Sequencing Project (2005). The map-based sequence of the rice genome. Nature 436, 793-800. doi: 10.1038/nature03895

Jefferson, R. A., Kavanagh, T. A., and Bevan, M. W. (1987). GUS fusions: betaglucuronidase as a sensitive and versatile gene fusion marker in higher plants. EMBO J. 6, 3901-3907.

Jung, K. H., An, G., and Ronald, P. C. (2008). Towards a better bowl of rice: assigning function to tens of thousands of rice genes. Nat. Rev. Genet. 9, 91-101. doi: $10.1038 / \mathrm{nrm} 2343$

Kang, H. G., Fang, Y., and Singh, K. B. (1999). A glucocorticoid-inducible transcription system causes severe growth defects in Arabidopsis and induces defense-related genes. Plant J. 20, 127-133. doi: 10.1046/j.1365-313X.1999. 00575.x

Khattri, A., Nandy, S., and Srivastava, V. (2011). Heat-inducible Cre-lox system for marker excision in transgenic rice. J. Biosci. 36, 37-42. doi: 10.1007/s12038011-9010-8

Kikuchi, S., Satoh, K., Nagata, T., Kawagashira, N., Doi, K., Kishimoto, N., et al. (2003). Collection, mapping, and annotation of over $28000 \mathrm{cDNA}$ clones from japonica rice. Science 301, 376-379. doi: 10.1126/science. 1081288
Kubo, M., Imai, A., Nishiyama, T., Ishikawa, M., Sato, Y., Kurata, T., et al. (2013). System for stable $\beta$-estradiol-inducible gene expression in the moss Physcomitrella patens. PLoS ONE 8:e77356. doi: 10.1371/journal.pone.0077356

Luo, K., Duan, H., Zhao, D., Zheng, X., Deng, W., Chen, Y., et al. (2007). 'GMgene-deletor': fused loxP-FRT recognition sequences dramatically improve the efficiency of FLP or CRE recombinase on transgene excision from pollen and seed of tobacco plants. Plant Biotechnol. J. 5, 263-274. doi: 10.1111/j.1467-7652. 2006.00237.x

Martinez, A., Sparks, C., Hart, C. A., Thompson, J., and Jepson, I. (1999). Ecdysone agonist inducible transcription in transgenic tobacco plants. Plant J. 19, 97-106. doi: 10.1046/j.1365-313X.1999.00504.X

McKenzie, M. J., Mett, V., Reynolds, P. H. S., and Jameson, P. E. (1998). Controlled cytokinin production in transgenic tobacco using a copper-inducible promoter. Plant Physiol. 116, 969-977. doi: 10.1104/pp.116.3.969

Mett, V. L., Lochhead, L. P., and Reynolds, P. H. S. (1993). Copper-controllable gene expression system for whole plants. Proc. Natl. Acad. Sci. U.S.A. 90, 4567-4571. doi: 10.1073/pnas.90.10.4567

Moore, I., Samalova, M., and Kurup, S. (2006). Transactivated and chemically inducible gene expression in plants. Plant J. 45, 651-683. doi: 10.1111/j.1365313X.2006.02660.x

Murray, M. G., and Thompson, W. F. (1980). Rapid isolation of high molecular weight plant DNA. Nucleic Acids Res. 8, 4321-4325. doi: 10.1093/nar/8.19.4321

Nandy, S., and Srivastava, V. (2012). Marker-free site-specific gene integration in rice based on the use of two recombination systems. Plant Biotechnol. J. 10, 904-912. doi: 10.1111/j.1467-7652.2012.00715.x

Odell, J. T., Nagy, F., and Chua, N. H. (1985). Identification of DNA sequences required for activity of the cauliflower mosaic virus $35 \mathrm{~S}$ promoter. Nature 313 , 810-812. doi: 10.1038/313810a0

Okuzaki, A., Konagaya, K., Nanasato, Y., Tsuda, M., and Tabei, Y. (2011). Estrogeninducible GFP expression patterns in rice (Oryza sativa L.). Plant Cell Rep. 30, 529-538. doi: 10.1007/s00299-010-0963-0

Ouwerkerk, P. B., de Kam, R. J., Hoge, J. H., and Meijer, A. H. (2001). Glucocorticoid-inducible gene expression in rice. Planta 213, 370-378. doi: 10.1007/s004250100583

Salter, M. G., Paine, J. A., Riddell, K. V., Jepson, I., Greenland, A. J., Caddick, M. X., et al. (1998). Characterisation of the ethanol-inducible alc gene expression system for transgenic plants. Plant J. 16, 127-132. doi: 10.1046/j.1365-313x. 1998.00281.x

Sambrook, J., and Russell, D. (2001). Molecular Cloning: A Laboratory Manual, 3rd Edn. Cold Spring Harbor, NY: Cold Spring Harbor Laboratory Press.

Sanders, P. R., Winter, J. A., Barnason, A. R., Rogers, S. G., and Fraley, R. T. (1987). Comparison of cauliflower mosaic virus $35 \mathrm{~S}$ and nopaline synthase promoters in transgenic plants. Nucleic Acids Res. 15, 1543-1558. doi: 10.1093/nar/15.4. 1543

Schena, M., Lloyd, A. M., Walbot, V., and Davis, R. W. (1991). A steroidinducible gene expression system for plant cells. Proc. Natl. Acad. Sci. U.S.A. 88, 10421-10425. doi: 10.1073/pnas.88.23.10421

Schlücking, K., Edel, K. H., Köster, P., Drerup, M. M., Eckert, C., Steinhorst, L., et al. (2013). A new $\beta$-estradiol-inducible vector set that facilitates easy construction and efficient expression of transgenes reveals CBL3-dependent cytoplasm to tonoplast translocation of CIPK5. Mol. Plant 6, 1814-1829. doi: 10.1093/mp/ sst065

Sreekala, C., Wu, L., Gu, K., Wang, D., Tian, D., and Yin, Z. (2005). Excision of a selectable marker in transgenic rice (Oryza sativa L.) using a chemically regulated Cre/loxP system. Plant Cell Rep. 24, 86-94. doi: 10.1007/s00299-0040909-5

Wang, X., Jiang, D., and Yang, D. (2015). Fast-tracking determination of homozygous transgenic lines and transgene stacking using a reliable quantitative real-time PCR assay. Appl. Biochem. Biotechnol. 175, 996-1006. doi: 10.1007/s12010-014-1322-3

Weinmann, P., Gossen, M., Hillen, W., Bujard, H., and Gatz, C. (1994). A chimeric transactivator allows tetracycline-responsive gene expression in whole plants. Plant J. 5, 559-569. doi: 10.1046/j.1365-313X.1994.5040559.x

Yoshida, K., Saitoh, H., Fujisawa, S., Kanzaki, H., Matsumura, H., Yoshida, K., et al. (2009). Association genetics reveals three novel avirulence genes from the rice blast fungal pathogen Magnaporthe oryzae. Plant Cell 21, 1573-1591. doi: 10.1105/tpc.109.066324 
Yu, J., Hu, S. N., Wang, J., Wong, G. K., Li, S., Liu, B., et al. (2002). A draft sequence of the rice genome (Oryza sativa L. ssp. Indica). Science 296, 79-92. doi: $10.1126 /$ science. 1068037

Zhang, S., Chen, L., and Goff, S. A. (2007). "Regulation of gene expression by small molecules in rice," in Novartis Foundation Symposium 236 - Rice Biotechnology: Improving Yield, Stress Tolerance and Grain Quality, eds J. A. Goode and D. Chadwick (Chichester: John Wiley \& Sons), 85-96. doi: 10.1002/ 9780470515778.ch7

Zhang, W., Subbarao, S., Addae, P., Shen, A., Armstrong, C., Peschke, V., et al. (2003). Cre/lox-mediated marker gene excision in transgenic maize (Zea mays L.) plants. Theor. Appl. Genet. 107, 1157-1168. doi: 10.1007/s00122-0031368-z

Zuo, J., Niu, Q. W., and Chua, N. H. (2000). An estrogen receptor-based transactivator XVE mediates highly inducible gene expression in transgenic plants. Plant J. 24, 265-273. doi: 10.1046/j.1365-313x.2000.00868.x
Zuo, J., Niu, Q. W., Møller, S. G., and Chua, N. H. (2001). Chemical-regulated, site-specific DNA excision in transgenic plants. Nat. Biotechnol. 19, 157-161. doi: $10.1038 / 84428$

Conflict of Interest Statement: The authors declare that the research was conducted in the absence of any commercial or financial relationships that could be construed as a potential conflict of interest.

Copyright (c) 2017 Chen, Cheng, Hu, Guo, Chen, Lin, Hu, Bellizzi, Lu, Wang, Wang, Chen and Wang. This is an open-access article distributed under the terms of the Creative Commons Attribution License (CC BY). The use, distribution or reproduction in other forums is permitted, provided the original author(s) or licensor are credited and that the original publication in this journal is cited, in accordance with accepted academic practice. No use, distribution or reproduction is permitted which does not comply with these terms. 